\title{
Multi-level heuristic to optimize the chemotherapy production and delivery
}

\author{
Alexis Robbes, Yannick Kergosien, Jean-Charles Billaut
}

\begin{abstract}
The bio pharmaceutical unit of Oncology Clinic (UBCO) of the hospital of Tours (France) produces between 100 and 300 injections per day for three hospital units of Tours. The production of chemotherapy drugs consists of two steps: a sterilization step and a preparation step performed by pharmacists. The production process can be modeled as an hybrid flow shop scheduling problem. Once the drugs are completed, they have to be delivered to the patient at a given due date. The delivery problem is a variant of the multi-trip vehicle routing problem. We propose in this paper a multi-level heuristic to solve the integrated production and delivery problem. Computational experiments are conducted on real-life based instances to compare multiple settings and to evaluate the efficiency of the proposed approach.
\end{abstract}

\section{Introduction}

The health care system is a demanding public service with various challenges. This paper focuses on an integrated chemotherapy production and delivery problem. In 2010, a first work with the bio pharmaceutical unit of Oncology Clinic (UBCO) of the hospital of Tours (France) [1] proposed to optimize the preparation of the chemotherapy products by solving a parallel machine scheduling problem. A first integrated solution to the UBCO [2] was presented in 2011. A method for a combined transportation and scheduling version of the problem [3] was proposed in 2017, however this study considers only one delivery man and a simplified workshop configuration. This paper is an extension of this study where the scheduling problem is an hybrid flow shop scheduling problem and the delivery problem is a variant of the Multi-trip vehicle Routing Problem. Even if the two sub-problems (the scheduling problem and the delivery problem) are considered independently, their

Alexis Robbes · Yannick Kergosien · Jean-Charles Billaut

Université de Tours, LIFAT EA 6300, CNRS, ROOT ERL CNRS 7002, 64 avenue Jean Portalis, 37200 Tours. e-mail: \{alexis.robbes, yannick.kergosien, jean-charles.billaut\}@univ-tours.fr 
resolution remains difficult. Most of the variants of the hybrid flow shop scheduling problem are NP-Hard [4], the same for the Multi-trip vehicle routing problem $[5,6]$. Integrated production and distribution scheduling problems have been studied in several papers $[7,8,9]$ where the objective functions are to minimize the combination of production and delivery costs or to minimize the makespan. In this study, we propose a model of an integrated chemotherapy drugs production and delivery problem which represents the real-life case (note that many services like UBCO have the same configuration). The objective is to minimize the total tardiness in order to provide a better health service quality. We propose a multi-level heuristic to solve the problem within a reasonable computation time, in order to be applied online and to compute an updated solution every time a new event occurs (e.g. the arrival of a new request).

\section{Problem definition}

The process of a chemotherapy treatment requires various steps. First of all the patient receives a medical consultation few days before the treatment. At the end of this consultation, the doctor prescribes the forthcoming treatment. A production order with the prescribed drugs is sent to the UBCO and another consultation is scheduled just before the beginning of the treatment. During this second consultation, the doctor checks the patient health and validates the previous prescription. The preparation of the order by the UBCO can only start after this validation in order to avoid the losses of drugs. Fig. 1 illustrates this process.

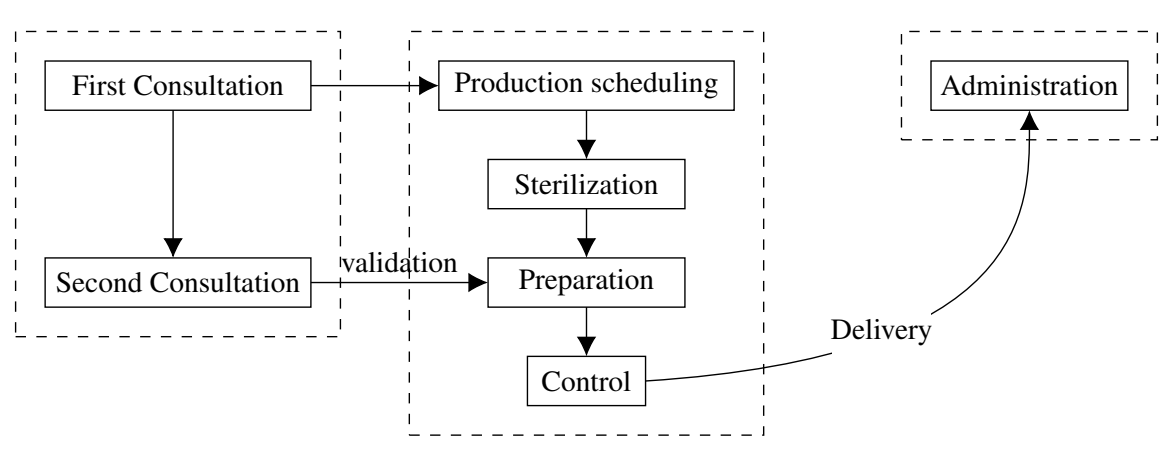

Fig. 1 Chemotherapy treatment process

The chemotherapy production is a 3-step process: Sterilization, Preparation and Control. Due to the isolators design, the Sterilization and the Preparation steps are done in the same isolator. Each isolator has several work stations where operators handmake the Preparation step. When a chemotherapy is completed, a Control step is executed on a single automated analyzer. 
The delivery part is done by a delivery men team. Each chemotherapy drug has to be delivered to a given patient. All patients are dispatched in different oncology units of several hospital units.

The set of chemotherapy drugs to produce and to deliver is represented by the set of jobs $J$. Each job $j$ in $J$ has a release date $r_{j}$ corresponding to the validation time before which the Preparation step cannot start, a processing time $p_{j}^{O}$ for the Preparation step, an assigned oncology unit $u_{j}$ where it has to be delivered before its due date $d_{j}$.

The production is done with $|I|$ identical parallel isolators. An isolator is characterized by a sterilizer capacity $Q$ (i.e maximum number of jobs), a Sterilization processing time $p^{S}$ (which do not depend on the sterilized batch of jobs) and a number of operators $m$ which can work at the same time (i.e. number of work stations).

The Control step is proceeded by a single automated analyzer. The Control processing time $p^{A}$ is the same for every job. To deliver the jobs, $|V|$ delivery men can make more than one trip. The objective function is to minimize the total tardiness $\sum_{j \in J} T_{j}$ where $T_{j}$ is the delivery tardiness of the job $j$ computed by $T_{j}=\max \left(0, D_{j}-d_{j}\right)$ where $D_{j}$ denotes the delivery date.

We propose a modelization of the chemotherapy production and delivery problem as an integrated scheduling and routing problem. The scheduling part corresponds to a 3-stage Hybrid Flow shop scheduling problem. The routing part corresponds to a variant of the Multi-Trip Vehicle Routing Problem with due dates.

Let consider a given schedule and a given delivery plan, for every job $j, c_{j}^{O}$ denotes the completion time of the Preparation step, $c_{j}^{A}$ denotes the completion time of the Control step. The batch Control completion time is the maximum Control completion time of the jobs in the batch.

Fig. 2 is a Gantt chart representing a partial solution of a problem instance with 2 isolators, 2 operators per isolator and 2 delivery men. As an example we highlight the process of the job 20 from the Sterilization step to the delivery. First, the job is sterilized in the first batch of isolator 1 . Then, it is prepared by operator 1 after its release date $r_{20}$ and after the end of the Sterilization step. This job is packed in a delivery batch with jobs 1 and 4 . This delivery batch is completed at $\max _{j \in\{1,4,20\}}\left(c_{j}^{A}\right)$ and is delivered by delivery man 1 . The delivery trip is the first one of delivery man 1 . The delivery man leaves the UBCO after the delivery batch completion time, then delivers jobs 1 and 4 at their assigned oncology unit $\left(u_{1}=u_{4}\right)$. The job 20 is then delivered at its oncology unit $u_{20}$. Finally, the delivery man comes back at the UBCO and is available for another trip. 

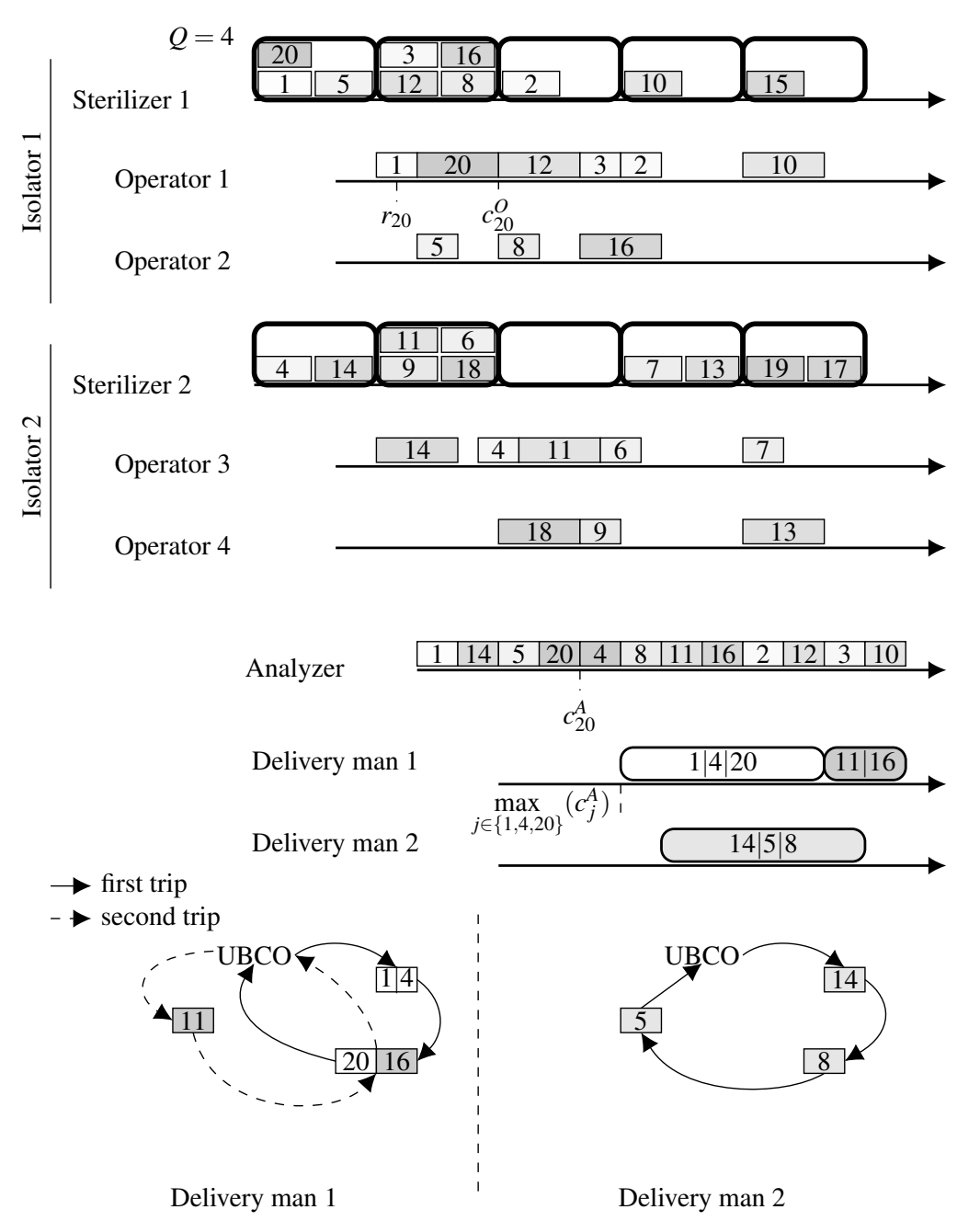

Fig. 2 Illustration of an instance of the problem: 2 isolators, 2 operators per isolator, 2 delivery men

\section{Lower Bound}

In order to propose a lower bound, we introduce revised release dates $\widetilde{r_{j}}=r_{j}+$ $p_{j}^{O}$ and revised due dates $\widetilde{d}_{j}=d_{j}-t_{0, j}$ where $t_{0, j}$ represents the shortest possible transportation time to deliver the job $j . \widetilde{r}_{j}$ is the minimum possible value for $c_{j}^{O}$ and $\tilde{d}_{j}$ is the maximum possible value for $c_{j}^{A}$ to deliver the job without tardiness.

Let consider the single machine scheduling problem with revised release dates, revised due dates, identical processing times $\left(p_{j}=p^{A}\right)$ and total tardiness minimiza- 
tion, which can be denoted by $1\left|r_{j}, p_{j}=p\right| \sum T_{j}$ using the 3-field Graham notation of scheduling problems [10]. Any lower bound of this problem is a lower bound of our problem. Indeed, the $1\left|r_{j}=\widetilde{r_{j}}, p_{j}=p^{A}, d_{j}=\widetilde{d}_{j}\right| \sum T_{j}$ problem is equivalent to our problem considering a large number of operators and delivery men.

The proposed lower bound is computed as follows. We build a pseudo instance where job $k$ has the $k$ th shortest revised release date $\widetilde{r}_{[k]}$ and the $k$ th shortest revised due date $\widetilde{d}_{[k]}$. The lower bound is given by the evaluation of sequence $(1, \ldots,|J|)$ of this pseudo instance.(Fig. 3 represents an instance with $\mathrm{J}=3$ jobs).

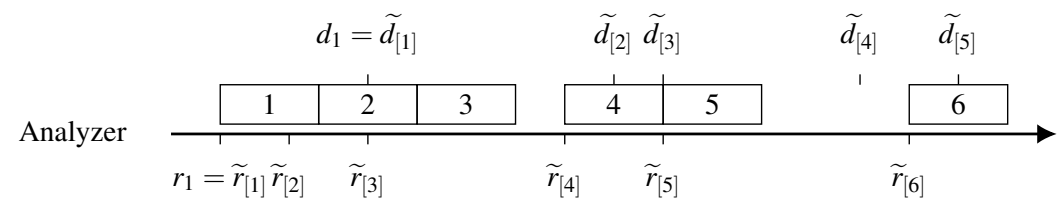

Fig. 3 Illustration of the Lower bound definition

\section{Multi-level heuristic}

The proposed multi-level heuristic is a constructive heuristic with multi-level decisions. The first decision level is the clustering of the jobs into delivery batches, the second decision level is the job assignment to a Sterilization batch, the third decision level is the Preparation scheduling, the fourth decision level is the Control scheduling and the last decision level is the delivery routing. Fig. 4 represents the flow chart of the multi-level heuristic.

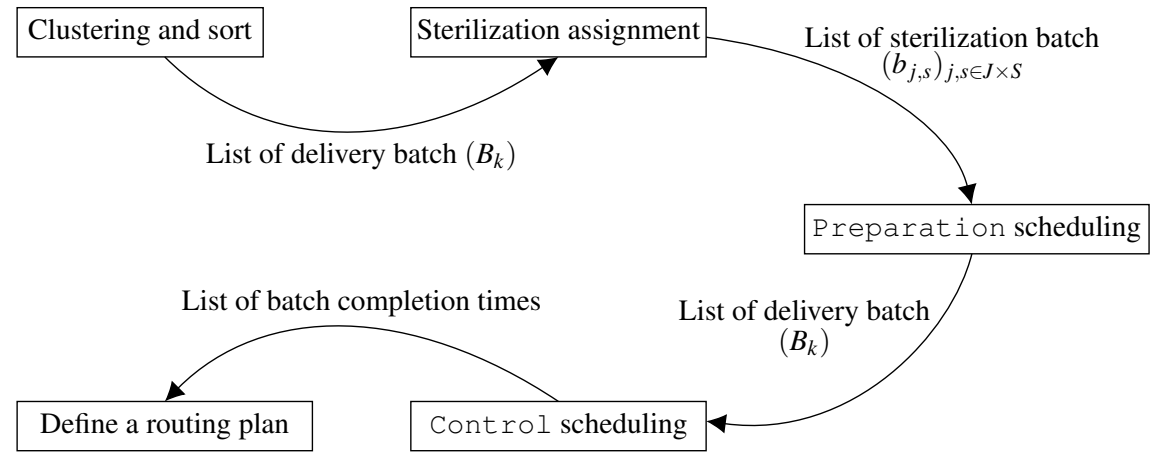

Fig. 4 Multi-level heuristic flowchart 
Clustering and sort: Each job is assigned to a cluster corresponding to a delivery batch by an Agglomerative Hierarchical Clustering method. This method needs a distance function between two clusters (i.e. batches). We define the distance between two batches $B$ and $B^{\prime}$ as the maximum Euclidean distance between the jobs which is named "complete link" in [11]: $\operatorname{dist}\left(B, B^{\prime}\right)=\max _{\left(j, j^{\prime}\right) \in B \times B^{\prime}}\left(\operatorname{dist}\left(j, j^{\prime}\right)\right)$. The Euclidean distance between jobs uses three dimensions: the oncology unit $u_{j}$ location, the due date $d_{j}$ and the revised release date $\widetilde{r}_{j}$. This clustering method aims to limit the waiting times before the delivery of each jobs and to reduce the duration of trips.

The number of delivery batches is given as an input. It is an important setting which is related to the maximum acceptable distance between two jobs from a same delivery batch.

The resulting delivery batches are then sorted using one sorting rule based on the due date of jobs of each batch. We propose three sorting rules by increasing order of:

1. $\min _{j \in B}\left(d_{j}\right)$, denoted MIN

2. mean $_{j \in B}\left(d_{j}\right)$, denoted MEAN

3. $\operatorname{median}_{j \in B}\left(d_{j}\right)$, denoted MED

A study of the impact of the number of delivery batches and the sorting rule is presented in Section 5.2.

Sterilization assignment: The sterilization assignment is an iterative method that consist in assigning jobs one by one to a sterilization batch. The jobs are sorted according to the sequence of delivery batches first (sorted by the selected sorting rule), then they are sorted in each delivery batch in $r_{j}+p_{j}^{O}$ increasing order. Then, each job is assigned to the last unfilled sterilization batch ending before the job's release date. If no such batch exists, the job is assigned to the first unfilled sterilization batch after the job's release date. In case of equality (i.e. two sterilizations batches complete at the same time) the job is assigned to the sterilization batch with the minimum sum of processing times $p_{j}^{O}$ of jobs already assigned to that batch. This sterilization batch assignment allocates the jobs to an isolator and its set of operators.

Preparation scheduling: For each isolator the jobs of the sterilization batches are successively scheduled. All the jobs of a sterilization batch must been scheduled before starting to schedule the jobs of the next sterilization batch. The jobs of each sterilization batch are sorted according to the sequence of delivery batches first, then they are sorted by increasing release date $r_{j}$. The jobs are then scheduled as soon as possible on the first available operator.

Control scheduling: The jobs are sorted first by increasing preparation completion time $c_{j}^{O}$ and in case of equality they are sorted according to the sequence of delivery batches. The jobs are successively scheduled as soon as possible. 
Routing: Delivery batches are assigned iteratively to the first available delivery man. The trip of a delivery batch is constructed by the Nearest Neighbor heuristic [12]. The delivery man repeatedly delivers to the nearest oncology units until all jobs have been delivered.

\section{Computational experiments}

In this section, the performances of the multi-level heuristic with various settings is evaluated on pseudo real life instances. We compare the proposed multi-level heuristic with the following 4-step method currently used at the UBCO:

1. Preparation: schedule the jobs by earliest release date first on the first available operator.

2. Control: schedule the jobs by earliest Preparation completion time first.

3. The clustering of jobs is done by the clustering algorithm described before with the following dimensions: Control completion times, due dates and oncology units.

4. The routes are defined by the Nearest Neighbor heuristic.

Note that the steps 3 and 4 are an approximation of the real life behavior of the delivery men who build their trips. This method is similar to a two-phase algorithm (scheduling then routing). This algorithm is called the Reference algorithm. The algorithms are implemented in Python language. Tests have been performed on an Intel(R) Core(TM) i5-7440HQ CPU @ 2.80GHz with 16 Go of Ram. The computation time of the two algorithms is about 1 second, which is acceptable for online use.

\subsection{Datasets}

The generation of 100 instances is inspired by the real case of the UBCO and have the following features:

- number of chemotherapies drugs: $|J|=150$

- due dates horizon: $\forall j \in J, d_{j} \in[9 \mathrm{~h}, 18 \mathrm{~h}]$

- release dates horizon: $\forall j \in J, r_{j} \in\left[d_{j}-10 \mathrm{~h}, d_{j}-50 \mathrm{~min}\right]$

- number of isolators: $|I|=4$

- production hours: [8h, 18h]

- Sterilization processing time: 15 minutes

- number of operators per isolator: 2 operators

- Preparation processing time: $p_{j}^{O} \in[5,15]$ minutes

- Control processing time: $p^{A}=5$ minutes

- number of oncology units: 60 units 
- number of delivery men: $|V|=3$

- all oncology units are within 35 minutes from the UBCO

For each interval, the distribution is uniform.

\subsection{Parameters setting and evaluation}

The number of delivery batches is a parameter that must be determined to find a good compromise:

- few delivery batches would result in long waiting times for the delivery men due to the time required to complete the batches

- a large number of delivery batches will generate a huge number of round trips which would imply waiting times for the completed batches.

To find the best compromise, we tested different number of delivery batches (from 15 to 35 ) on the 100 instances.

For each instance we determined the gap between the lower bound and the solution found. The gap is computed as $g a p=\frac{h-L b}{L b}$ where $h$ is the total tardiness found by the proposed multi-level heuristic or the Reference algorithm and $L b$ is the lower bound defined in Section 3.

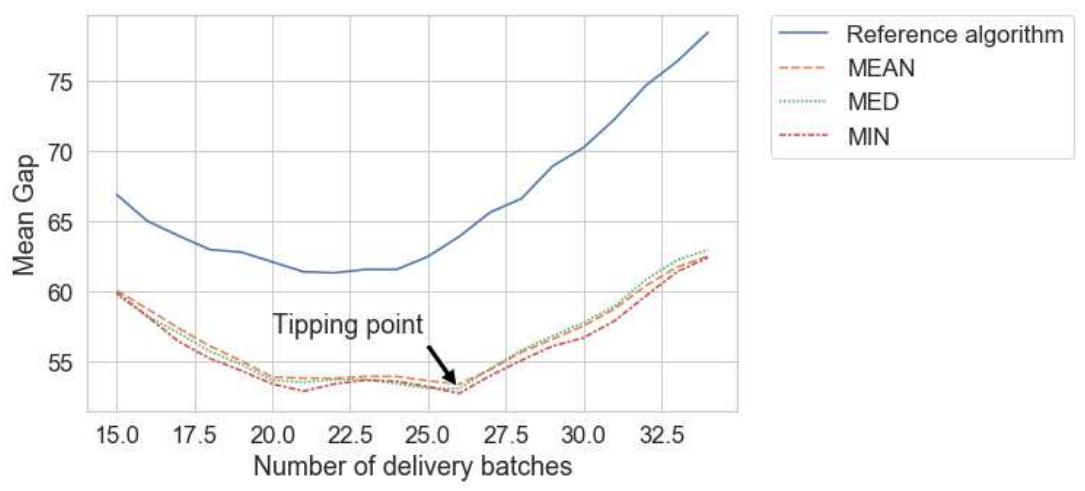

Fig. 5 Impact of the number of delivery batches - Mean Gap

Fig. 5 represents the evolution of the mean gap for each tested number of delivery batches and for each sorting rule. Fig. 5 shows that increasing the number of delivery batches increases the quality until a tipping point around 26 for the proposed multilevel heuristic and 24 for the Reference algorithm.

A delivery of 150 chemotherapy drugs in 26 trips means an average delivery batch size of 6 jobs and just under three trips per hour.

The best average gap found is around $52 \%$ which seems to be a big value. However, the lower bound, defined in section 3 , is clearly weak but is useful to compare 
the methods with a common reference. The lower bound weakness is mostly due to the assumption of an infinite number delivery men. It seems that the choice of the sorting rule do not have a big impact on the mean gap.
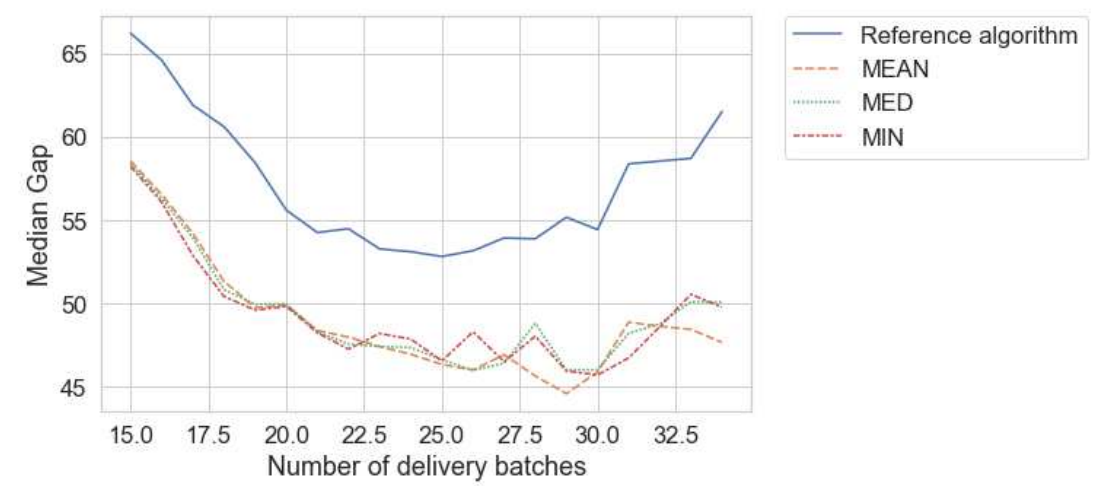

Fig. 6 Impact of the number of delivery batches - Median Gap

Fig. 6 presents the same results as Fig. 5 using the median gap instead of the mean gap. Around the tipping point, we note that the MEAN presents the best results on the median gap whereas there is not much difference between the three rules on the mean gap.

The median gap is smaller than the mean gap. One of the reason would be the existence of few outliers.

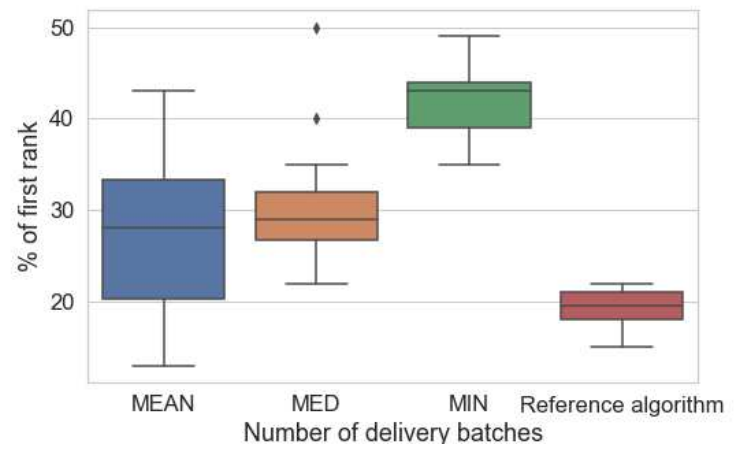

Fig. 7 Boxplots of the percentage of been the best heuristic i.e. first rank (number of delivery batches varying between 15 and 35)

Fig. 7 illustrates the quality difference of the sorting rules. The size of the boxplots shows that the Reference algorithm is the best algorithm around $20 \%$ of the 
time without depending of the number of delivery batches. However, it is the MIN rule which is the best most of the time (around 45\%). This is in accordance with Fig. 5.

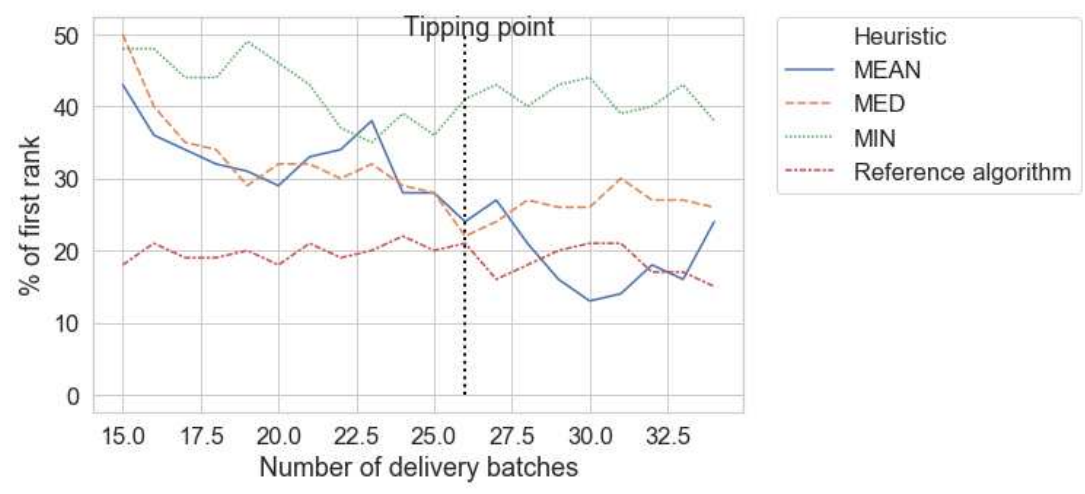

Fig. 8 Percentage of times a heuristic gives the best result i.e. first rank

Fig. 8 illustrates the influence of the number of delivery batches on the heuristics ranking. We can see that the MIN rule is more is often the best heuristic whatever the number of delivery batches. While, the MEAN rule ranking is really dependent of the number of delivery batches.

\section{Conclusions and future works}

A real case of production and delivery of chemotherapy drugs was studied and a model of the problem was proposed. The model is based on an integrated version of a hybrid flowshop scheduling problem and a multi-trip vehicle routing problem. To quickly solve the problem, we proposed a multi-level heuristic which schedules the production after taking into account the delivery part. The numerical experiments showed the efficiency of the proposed method compared to a Reference algorithm corresponding to the current planning method. A study of the multi-level settings illustrated the importance of the number of delivery batches and of the sorting rule.

Several research perspectives can be considered. First, the lower bound quality may be improved on the routing part. Besides, a local search at each scheduling level could improve the quality of the heuristic. 


\section{References}

1. Alexandre Mazier, Jean-Charles Billaut and Jean-François Tournamille. Scheduling preparation of doses for a chemotherapy service. Annals of Operations Research (2010).

2. Yannick Kergosien, Jean-François Tournamille, Brunos Laurence and Jean-Charles Billaut. Planning and tracking chemotherapy production for cancer treatment: A performing and integrated solution. International Journal of Medical Informatics (2011).

3. Yannick Kergosien, M. Gendreau and Jean-Charles Billaut. Benders decomposition based heuristic for a production and outbound distribution scheduling problem with strict delivery constraints. European Journal of Operational Research 262 (1), 287-298 (2017).

4. Farzaneh Abyaneh and Saeedeh Gholami. A comparison of algorithms for minimizing the sum of earliness and tardiness in hybrid flow-shop scheduling problem with unrelated parallel machines and sequence-dependent setup times. Journal of Industrial and Systems Engineering 8 (2), 67-85 (2015).

5. Diego Cattaruzza and Nabil Absi and Dominique Feillet. The Multi-Trip Vehicle Routing Problem with Time Windows and Release Dates. Transportation Science 50 (2) , 676-693 (2016).

6. Nabila Azi, Michel Gendreau and Jean-Yves Potvin. Optimization and Approximation in Deterministic Sequencing and Scheduling: a Survey. European Journal of Operational Research, 202 (3), 756-763 (2010).

7. P. Amorim, M.A.F. Belo-Filho, F.M.B. Toledo, C. Almeder and B. Almada-Lobo. Lot sizing versus batching in the production and distribution planning of perishable goods. International Journal of Production Economics 146 (1), 208-218 (2013).

8. M.A.F. Belo-Filho, P. Amorim and B. Almada-Lobo. An adaptive large neighbourhood search for the operational integrated production and distribution problem of perishable products. International Journal of Production Research 53 (20), 6040-6058 (2015).

9. Priyantha Devapriya, William Ferrell and Neil Geismar. Integrated production and distribution scheduling with a perishable product. European Journal of Operational Research 259 (3), 906-916 (2017).

10. R.L. Graham, E.L. Lawler, J.K. Lenstra and A.H.G.Rinnooy Kan. An exact algorithm for a vehicle routing problem with time windows and multiple use of vehicles. Discrete Optimization II, 5, 287-326 (1979).

11. Fionn Murtagh. A Survey of Recent Advances in Hierarchical Clustering Algorithms. The Computer Journal 26 (4), 354-359 (1983).

12. Daniel J. Rosenkrantz, Richard E. Stearns and Philip M. Lewis An Analysis of Several Heuristics for the Traveling Salesman Problem. SIAM Journal on Computing 6 (3), 563-581 (1977). 